\title{
Contribuciones de Zamorano al Entendimiento de la Biodiversidad de los Artrópodos de Honduras
}

\author{
Oliver Schlein ${ }^{1}$ y Ronald D. Cave ${ }^{2}$
}

Resumen. La Colección de Artrópodos de Zamorano fue fundada hace más de 30 años y tiene más de 250,000 especímenes, los órdenes más dominantes son Coleoptera, Hymenoptera y Lepidoptera. Más de 90,000 especímenes identificados están digitalizados en su propia base de datos y han sido recolectados en más de 300 sitios, la mayoría en Honduras. Los inventarios de escarabajos de las familias Cerambycidae y Scarabaeidae y de parasitoides himenópteros son de valor científico enorme y único en toda la región. Cuatro proyectos muy influyentes, financiados por fondos externos, fueron realizados durante los primeros 25 años de la colección y están descritos con sus logros centrales: 1) inventario de los parasitoides de la mosca blanca de la batata, Bemisia tabaci (Gennadius); 2) la diversidad y biología de los escarabajos joya, Chrysina spp. (Scarabaeidae); 3) inventario de la subfamilia Dynastinae (Scarabaeidae); y 4) la capacitación de maestros, guardarecursos y guías sobre varios aspectos de la entomología. Catorce estudiantes zamoranos hicieron sus tesis en biodiversidad vinculadas con la misión de la Colección. Zamorano apoya la organización, la logística y las investigaciones entomológicas internacionales en Honduras. Además, en cooperación con fundaciones e instituciones nacionales y estatales, se investiga la biodiversidad de artrópodos en áreas protegidas de Honduras. En la gran mayoría de estas áreas, pocos o ningún dato existe sobre la fauna de artrópodos presente. Próximamente, se unirá la Colección de Artrópodos de Zamorano con otra colección importante regionalmente, el Museo Entomológico de León, Nicaragua, añadiendo otros 250,000 especímenes, en su mayoría recolectados en Nicaragua, para ser incorporados al inventario presente. Así, la colección de Zamorano contará con aproximadamente 500,000 especímenes y se convertirá en la colección más grande y representativa de Honduras y Nicaragua, y será una de las colecciones más grandes e importantes de Centroamérica.

Palabras clave: Áreas protegidas, entomología, inventarios de especies, proyectos internacionales, Scarabaeidae, taxonomía.

\section{Zamorano's Contributions to the Understanding of Arthropod Biodiversity in Honduras}

\begin{abstract}
The Zamorano Arthropod Collection was founded more than 30 years ago. It includes more than 250,000 specimens, with the dominant orders being Coleoptera, Hymenoptera, and Lepidoptera. Data of more than 90,000 of the identified insect specimens are digitized in a comprehensive database, and were collected in more than 300 sites, most within Honduras. The inventories of scarab and longhorn beetles, as well as of hymenopterous parasitoids, are of outstanding scientific value and unique in the region. Four influential projects, financed by external funds, were performed during the first 25 years of the collection's existence, and are described with their main achievements: 1) inventory of the parasitoids of the sweetpotato whitefly, Bemisia tabaci (Gennadius); 2) diversity and biology of jewel scarab Chrysina spp. (Scarabaeidae); 3) inventory of the scarab beetle subfamily Dynastinae (Scarabaeidae); and 4) advanced capacity-building for teachers, nature reserve guards, and guides in various aspects of entomology. Often, support is provided in the way of coordination, logistics, and implementation for foreign entomological research projects in Honduras. Also, in cooperation with national foundations and governmental institutions, research on the arthropod biodiversity of protected areas in Honduras is conducted. For most of these, only fragmentary or no data still exist concerning the present arthropod fauna. During the coming years, the Zamorano Arthropod Collection will be united with another collection of outstanding regional importance, The Entomological Museum currently located in León, Nicaragua; this will add another 250,000 specimens, mainly collected in Nicaragua, to the Zamorano collection. Therefore, the Zamorano collection will house about 500,000 insect specimens and will evolve into the most comprehensive and representative arthropod collection of both Honduras and Nicaragua, becoming one of the largest and most important arthropod collections in Central America.
\end{abstract}

Keywords: Entomology, international projects, protected areas, Scarabaeidae, species inventories, taxonomy..

${ }^{1}$ Escuela Agrícola Panamericana, Zamorano, Departamento de Ambiente y Desarrollo, Apartado Postal No. 93, 11101 Tegucigalpa, Honduras, C.A. Email: oschlein@ zamorano.edu

${ }^{2}$ Indian River Research and Education Center, University of Florida, Department of Entomology and Nematology, 2199 South Rock Road, Fort Pierce, Florida, U.S.A. Email: rdcave@ufl.edu

DOI: $10.5377 /$ ceiba.v52i1.1036 


\section{Introducción}

La Colección de Artrópodos de Zamorano tiene más de 30 años de existencia y actualmente aloja más de 250,000 especímenes. La mayoría de los especímenes adultos pertenecen a los órdenes de escarabajos (Coleoptera), mariposas y polillas (Lepidoptera), y avispas (en su mayoría parasitoides), abejas y hormigas (Hymenoptera). Más de 90,000 especímenes están identificados y digitalizados en su propia base de datos, con más de 2,050 géneros representados; entre ellos se encuentran 323 especímenes tipos. Además, existe una colección de artrópodos (larvas de insectos, arácnidos, milpiés y ciempiés) y moluscos conservados en alcohol.

El entomólogo Dr. Ronald Cave, ahora en la Universidad de Florida, Estados Unidos (Figura 1) formó la colección de Zamorano, con su apoyo se incrementó la cantidad de especímenes enormemente. También, el Dr. Cave inició la base de datos de la colección durante su trabajo de 15 años en Zamorano. Desde octubre de 2008, el Dr. Oliver Schlein trabaja como entomólogo y curador de la colección en Zamorano. Los especímenes han sido recolectados en más de 300 lugares, la mayor parte en Honduras.

Científicos internacionales trabajan con los materiales de la colección para identificar especies de sus propias investigaciones. En cooperación con otros países, Zamorano coordina y apoya proyectos entomológicos que implican la recolección de insectos en regiones fuera de Honduras. Sin embargo, fuera de Zamorano, todavía son pocas las personas que conocen de la existencia de la Colección de Artrópodos de Zamorano, ni siquiera su importancia.

La colección tiene tres funciones. La primera es proveer material identificado por expertos, que se puede usar como referencia para facilitar la interpretación de la literatura usada para identificar géneros y especies. La segunda función es hacer disponible los mismos especímenes identificados por expertos para hacer identificaciones comparativas cuando la literatura para identificar no existe o no está a la mano. La tercera función es documentar la diversidad insectil en el país y hacer los especímenes y su información asociada disponibles a la comunidad científica. La importancia de mantener una colección de artrópodos radica en utilizar la información y elaborar los listados de las especies encontradas. Estos listados son el primer paso para conocer las especies existentes para conducir investigaciones en ecología, biogeografía, conservación, control biológico, manejo de plagas y entomología forense.

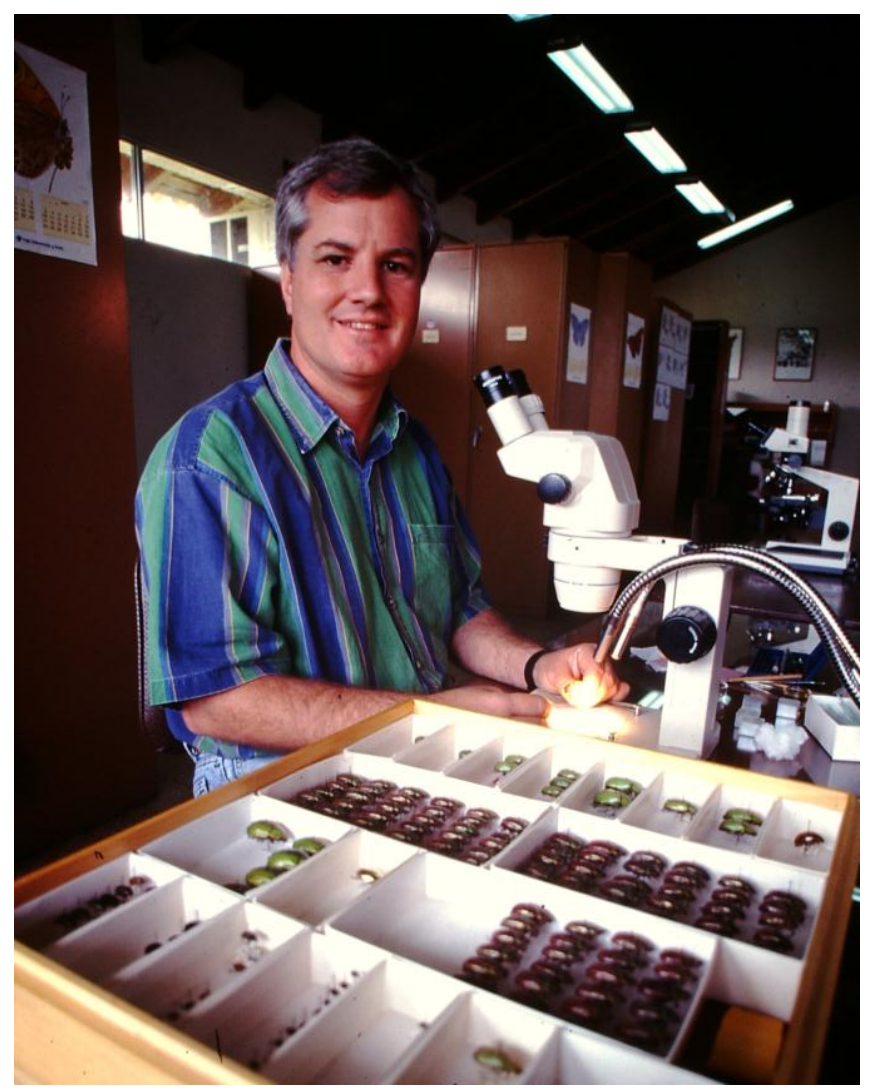

Figura 1. El entomológo Ronald D. Cave trabajando en la Colección de Artrópodos de Zamorano (c1996).

\section{La Colección de Artrópodos de Zamorano y Proyectos Centrales de los Primeros 25 Años}

La Colección de Artrópodos de Zamorano se inició en 1980 con pocos ejemplares de importancia agrícola. Desde 1980 hasta 1988, muchos especímenes de las colecciones que hacían los estudiantes en la clase de entomología se agregaron a la colección. De otros lugares, muchos especímenes fueron recolectados por el personal del proyecto Manejo Integrado de Plagas en Honduras (MIPH) dirigido por el Dr. Keith Andrews. En los años 80, en el proyecto 
MIPH se diagnosticaban daños y plagas en muchos cultivos como maíz, repollo y frijol. Hasta 1988, la colección se dividió en dos partes. Una parte incorporó los especímenes de las colecciones que hacían los estudiantes en la clase de entomología y fue organizada por orden y familia, pero no fueron identificados hsta género ni especie. Otra parte se organizó por cultivo, con algunos especímenes identificados hasta género y especie. La colección, antes conocida como Inventario Agroecológico, es ahora una de las colecciones más importantes de Honduras.
La colección de insectos en Zamorano llegó a desarrollarse por completo al llegar el Dr. Cave en 1987, marcando un gran crecimiento en el número de ejemplares y colectas en el país. En 1988, se terminó la construcción de la infraestructura donde está alojada la colección. Cave organizó la colección en forma taxonómica, como existe hoy. Desde 1988, la mayoría de los especímenes son de las recolecciones del Dr. Cave, personal trabajando bajo su supervisión (particularmente el Ing. Roberto Cordero y Julio Torres) y estudiantes que realizaron tesis en el área de biodiversidad de insectos (Cuadro 1).

Cuadro 1. Tesis de estudiantes de la Escuela Agrícola Panamericana, Zamorano, Honduras, que realizaron su investigación en la biodiversidad de insectos (1989-2012).

Acosta, N. 1992. Inventario y evaluación de enemigos naturales de Liriomyza spp. (Diptera: Agromyzidae) en la región sur de Honduras. Tesis Ing. Agr. Escuela Agrícola Panamericana, Zamorano, Honduras.

Argueta Avelar, G.E. 2002. Apidae en Honduras: Listado, distribución y biología de las especies. Tesis Ing. Agr. Escuela Agrícola Panamericana, Zamorano, Honduras.

Arias Roda, F. 2012. Refugios para enemigos naturales de plagas insectiles: Selección inicial de plantas para condiciones del Zamorano. Tesis Ing. Agr. Escuela Agrícola Panamericana, Zamorano, Honduras.

Arismendi Solís, N.L. 2002. Pentatomidae en Honduras: Listado, distribución y biología de las especies. Tesis Ing. Agr. Escuela Agrícola Panamericana, Zamorano, Honduras.

Collart Marves, C.E. 2010. Entomofauna del corredor biológico de uso múltiple Texiguat, El Paraíso, Honduras. Tesis Ing. Agr. Escuela Agrícola Panamericana, Zamorano, Honduras.

Cordero, R.J. 1989. Parasitoides de Plutella xylostella L. (Lepidoptera: Plutellidae) en cultivo de repollo en Honduras. Tesis Ing. Agr. Escuela Agrícola Panamericana, Zamorano, Honduras.

González, M. 1994. Dinámica poblacional y parasitismo de la mosca doméstica, Musca domestica, y la mosca del establo, Stomoxys calcitrans. Tesis Ing. Agr. Escuela Agrícola Panamericana, Zamorano, Honduras.

Granadino Urbina, C.A. 1992. Inventario de artrópodos y patógenos de anonaceas en Honduras. Tesis Ing. Agr. Escuela Agrícola Panamericana, Zamorano, Honduras.

Jut Solorzano, J.C. 2012. Comparación de la biodiversidad de escarabajos estercoleros (Scarabaeidae: Scarabaeinae) en diferentes ecosistemas en el Parque Nacional Nombre de Dios, Atlántida, Honduras. Tesis Ing. Agr. Escuela Agrícola Panamericana, Zamorano, Honduras.

Machado Caballero, J.E. 2001. Inventario y estudio comparativo de la fauna de Odonata en tres áreas de Honduras. Tesis Ing. Agr. Escuela Agrícola Panamericana, Zamorano, Honduras.

Márquez, J.G. 1990. Complejo de parasitoides de Coccidae, Diaspididae y Aleyrodidae en el cutivo de cítricos en Honduras. Tesis Ing. Agr. Escuela Agrícola Panamericana, Zamorano, Honduras.

Morjan Erazo, W.E. 1993. Depredadores nocturnos de plagas de maíz y de frijol en dos sistemas de labranza. Tesis Ing. Agr. Escuela Agrícola Panamericana, Zamorano, Honduras.

Peña Fonseca, G.A. 2001. Capacitación y concientización ambiental sobre insectos en dos áreas protegidas de Honduras. Tesis Ing. Agr. Escuela Agrícola Panamericana, Zamorano, Honduras.

Vélez Izaguirre, J.J. 1993. Relación entre la etapa fenológica y la variedad de frijol con el nivel de parasitismo de Bemisia tabaci Gennadius. Tesis Ing. Agr. Escuela Agrícola Panamericana, Zamorano, Honduras. 
Después de unos intentos con varios programas, Cave inició la presente base de datos de la colección en 1998. Esta base de datos usa el programa Orthoptera DB, desarrollado por P. Nasrecki y manejado por FileMaker Pro. Actualmente, la colección cuenta con más de 250,000 ejemplares de los cuales alrededor de 90,000 ejemplares están identificados y digitalizados en esta base de datos, han sido recolectados en más de 300 localidades, la mayoría en Honduras. La colección contiene más de 250 paratipos de 20 especies, ante todo de la familia Scarabaeidae (Coleoptera) y la superfamilia Chalcidoidea (Hymenoptera). Los órdenes dominantes en la colección de Zamorano son Coleoptera (más de 930 géneros), Hymenoptera y Lepidoptera (cada uno con más de 250 géneros identificados). Dentro del orden Coleoptera, por el trabajo intensivo del Dr. Cave y el Dr. Ratcliffe, la colección de las subfamilias Dynastinae y Rutelinae son inventarios de referencia únicos para Honduras y toda la región, además de un valor científico inestimable. Igualmente, el inventario de los escarabajos de la familia Cerambycidae (más de 190 géneros) es seguramente lo más completo y mejor identificado de toda Honduras.

Cuatro proyectos financiados externamente abrieron muchas oportunidades para viajar al campo y recolectar intensivamente en muchos agroecosistemas y áreas protegidas. El primero fue un proyecto financiado por el Programa Regional de Reforzamiento a la Investigación sobre los Granos Básicos en Centroamérica, de la Comunidad Económica Europea (1993-1994). Se realizó un inventario de los parasitoides de la mosca blanca de la batata, Bemisia tabaci (Gennadius) (Hemiptera: Aleyrodidae) como estudio preliminar para la implementación del control biológico de la mosca blanca con parasitoides. En este estudio se encontraron ocho especies de parasitoides que parasitan las ninfas de $B$. tabaci, y se detectó una especie de hiperparasitoide. Esta investigación fue importante para un proyecto en el cual se introdujo a Honduras una especie de Eretmocerus de India para el control biológico clásico.

A partir de 1994, varios científicos llegaron a Honduras para recolectar escarabajos en colaboración con Zamorano. Los escarabajos del género Chrysina (Coleoptera: Scarabaeidae) o escarabajos joyas fueron los más favorecidos (Figuras 2 y 3). Fue un poco difícil de obtener permisos de colectar porque hubo mucha preocupación de personas en el gobierno y la Universidad Nacional Autónoma de Honduras por el efecto que los colectores causarían en las poblaciones de Chrysina. Con el propósito de reducir estas preocupaciones y demostrar que las Chrysina no son raras y las poblaciones no están en peligro de extinción por la colecta para fines científicos, Cave y David Hawks (Universidad de California-Riverside) colaboraron en un proyecto financiado por la National Geographic Society (1997-1998).

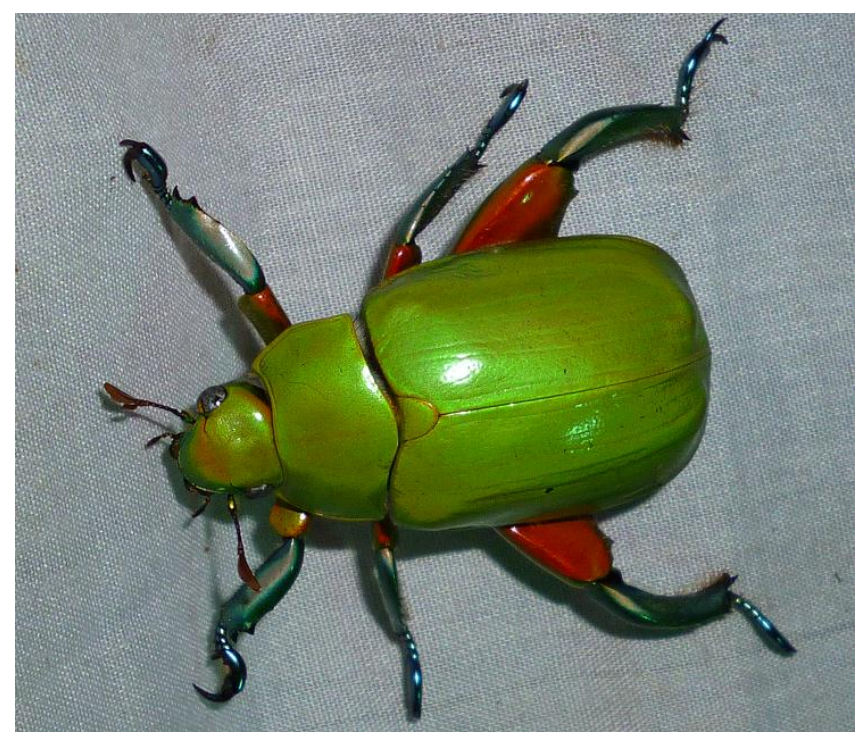

Figura 2. Macho del escarabajo joya Chrysina cavei (Scarabeidae: Rutelinae), una especie endémica de Honduras.

Los objetivos de este proyecto fueron (1) obtener información sobre la abundancia de las poblaciones de todas las especies de Chrysina en cuatro áreas protegidas (Parque Nacional La Muralla, Parque Nacional Pico Pijol, Parque Nacional Cusuco y la Reserva Biológica Güisayote), (2) determinar la efectividad de atracción de lámparas de vapor de mercurio y su potencial de sobre recolección de las poblaciones, (3) obtener datos sobre las características relativas de dispersión de estos escarabajos, (4) incrementar los conocimientos de sus distribuciones en Honduras, (5) identificar los árboles hospederos y otros factores ecológicos que determinan la 
distribución y (6) proveer la información recolectada al Departamento de Áreas Protegidas y Vida Silvestre de la Administración Forestal del Estado y a la Secretaría de Recursos Naturales y Ambiente de Honduras. Además del descubrimiento de la nueva especie Chrysina cavei Hawks \& Bruyea (Hawks y Bruyea, 1999), los viajes mensuales a las cuatro áreas protegidas permitieron recolectar muchos especímenes para la Colección de Artrópodos de Zamorano.

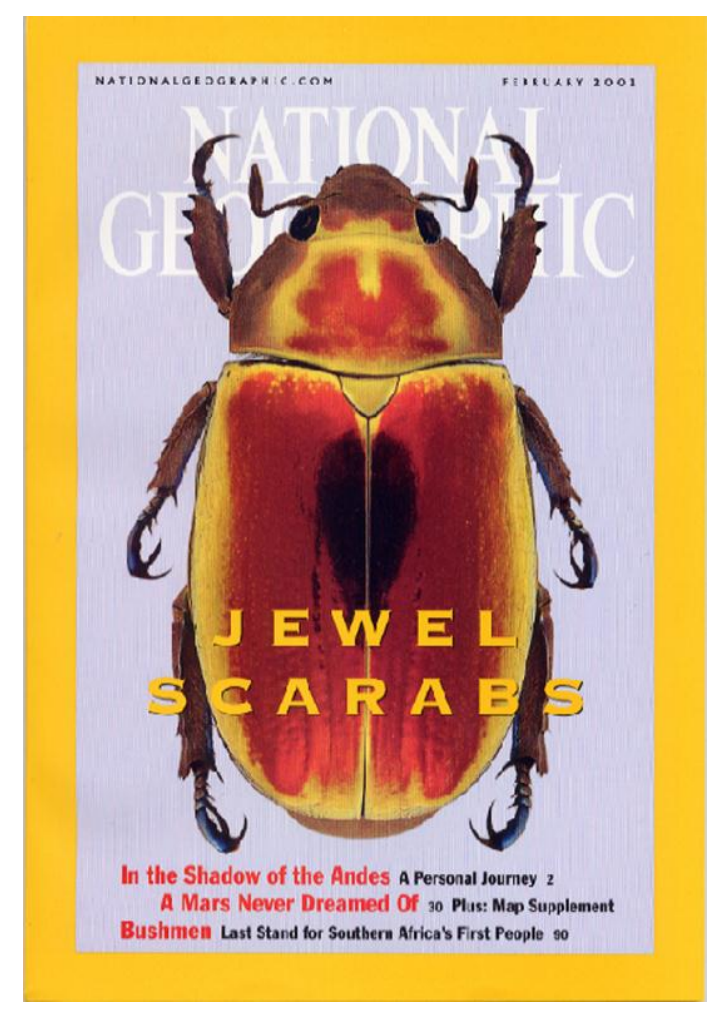

Figura 3. Portada de la publicación Cave, R.D. 2001. Jewel scarabs. National Geographic Magazine 199(2): 52-61.

El tercer proyecto aprovechó la colaboración con el Dr. Brett Ratcliffe (Universidad de Nebraska) para obtener financiamiento de la National Science Foundation (Estados Unidos) para un inventario faunístico de los Dynastinae (Coleoptera: Scarabaeidae) en Honduras, Nicaragua y El Salvador (1998-2002). Los objetivos del proyecto fueron (1) estudiar los ejemplares de Dynastinae que ya existen en las colecciones de Honduras, El Salvador, Nicaragua y Norte América, (2) recolectar intensivamente en numerosas localidades y diferentes épocas en Honduras, El Salvador y Nicaragua, (3) ensamblar y depositar en colecciones ejemplares identificados por autoridades y (4) publicar una monografía de los Dynastinae de Honduras, El Salvador y Nicaragua.

La monografía (Ratcliffe y Cave 2006) es una revisión comprehensiva de las 116 especies de escarabajos dinástinos que hay en Honduras, Nicaragua y El Salvador (Figura 4). Se describieron seis especies nuevas y se reportaron 14 nuevos registros de país para El Salvador, 32 nuevos registros de país para Honduras, y 26 nuevos registros de país para Nicaragua. El tomo incluye claves bilingües para todos los taxones, descripciones de todos los taxones, distribuciones geográficas y estacionales, datos ecológicos y biológicos, datos biogeográficos $\mathrm{y}$ numerosas ilustraciones.

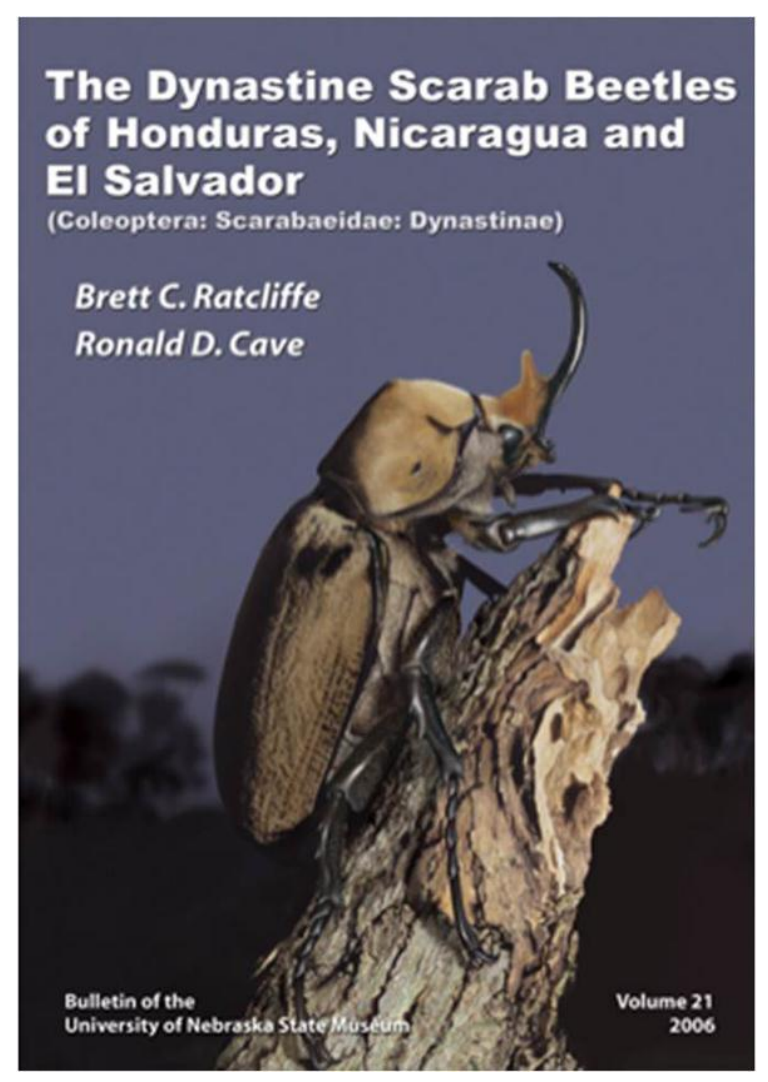

Figura 4. Portada de la publicación Ratcliffe, B.C. y R.D. Cave. 2006. The dynastine scarab beetles of Honduras, Nicaragua, and El Salvador. Bulletin of the University of Nebraska State Museum 21: 1-424 
El cuarto proyecto, Capacitación de Maestros, Guardarecursos y Guías Naturalistas e Inventario de Insectos en Dos Áreas Protegidas y sus Comunidades fue financiado por la Fundación VIDA y USAID (1999-2002). Realizó actividades para resolver en parte los problemas del desconocimiento de la biodiversidad y escasez de información, por medio de capacitación, asistencia técnica, preparación y divulgación de materiales educativos y exhibiciones, entrenamiento e investigación aplicada.

Los objetivos del proyecto fueron (1) capacitar maestros de escuelas, guardarecursos y guías naturalistas en la variabilidad de la biodiversidad de los insectos y su ecología, rol e importancia en los ecosistemas, (2) preparar y divulgar materiales educativos sobre la biodiversidad insectil y su ecología, rol e importancia en los ecosistemas y (3) inventariar los insectos en la Reserva de Vida Silvestre Cuero y Salado y el Parque Nacional Pico Bonito, además entrenar y equipar parataxónomos en las dos áreas protegidas. Un parataxónomo es una personas rural sin educación formal en biología, pero capacitada en la recolección e identificación de especímenes biológicos y capaz de trabajar independientemente y comprender la filosofía y valor de su trabajo.

Los logros de este proyecto cumplieron las metas generales de hacer las áreas protegidas experiencias de aprendizaje para las comunidades vecinas y sus visitantes y entender mejor la fauna de los insectos dentro de las áreas para que se conozcan y se aprovechen ecológica y económicamente los tesoros naturales dentro de sus bordes.

- Se entrenaron ocho guardarecursos y guías naturalistas en la identificación de insectos y plantas, la ecología básica de los artrópodos y las plantas y el rol e importancia de los artrópodos en los ecosistemas forestales.

- Se capacitaron 61 maestros de escuelas sobre la ecología e importancia de los artrópodos en los ecosistemas.

- Se publicó el libro La Vida Fascinante y Exitosa de los Insectos (Cave et al. 2002). El libro trata de la morfología, biología, ecología, comportamiento y diversidad de insectos, además, tiene más de 50 fotografías y dibujos de insectos.
- Se produjeron nueve afiches con fotografías de algunos de los insectos más espectaculares en Honduras. Los afiches destacaron los órdenes Lepidoptera, Coleoptera y Hemiptera, las familias Nymphalidae, Saturniidae, Scarabaeidae y Cerambycidae, y varias especies endémicas de Honduras.

- Se construyeron dos exhibiciones para mostrar información interesante a grupos escolares que visitan el Museo de Mariposas en La Ceiba y el Centro de Visitantes de la Reserva de Vida Silvestre Cuero y Salado. Cada exhibición muestra los órdenes principales de los insectos, el uso de los insectos por el hombre y los alimentos de los insectos

- Se produjo el video El Estudio de la Biodiversidad de Insectos, tiene una duración de 35 minutos y demuestra los métodos usados en el estudio de la biodiversidad de insectos.

- Se elaboraron tres colecciones sinópticas para mostrar la diversidad de insectos en Honduras. Cada colección contiene más de 800 especímenes. Una colección fue donada a la Fundación Cuero y Salado (FUCSA), una a la Fundación Parque Nacional Pico Bonito (FUPNAPIB) y una a la Fundación VIDA.

- Se elaboró la guía Insectos de Honduras (Cave y Cordero 2002) con 167 fotografías a color de 163 especies de insectos, y se donaron cientos de copias a FUCSA y FUPNAPIB.

- Se inició un inventario de los insectos en la Reserva de Vida Silvestre Cuero y Salado y el Parque Nacional Pico Bonito. Durante 25 viajes al campo se recolectaron más de 20,000 especímenes de insectos utilizando red, trampas, lonas y luces.

- Se entrenaron seis estudiantes universitarios en temas de desarrollo rural, trabajo con comunidades y gente rural, planificación de proyectos, investigación sobre algunas familias de insectos en Honduras, manejo de base de datos y manejo de una colección. Cuatro estudiantes (Cuadro 1: Machado Caballero 2001, Peña Fonseca 2002, Argueta Avelar 2002 y Arismendi Solís 2002) de Zamorano realizaron su tesis de grado dentro del contexto del proyecto. Dos estudiantes del Departamento de Biología de la 
Universidad Nacional Autónoma de Honduras (UNAH) colaboraron con el proyecto al realizar estudios relacionados con el inventario de insectos. Luis Soto finalizó su tesis Nymphalidae, Papilionidae y Pieridae de Honduras, y Ana Samayoa cumplió su tesis Sphingidae de Honduras (Samayoa y Cave, 2008).

\section{Colaboraciones y Proyectos Nacionales Desde el 2008}

En cooperación con el Instituto Nacional de Conservación y Desarrollo Forestal, Áreas Protegidas y Vida Silvestre (ICF), con fundaciones locales y varios científicos visitantes internacionales, y la Empresa Nacional de Energía Eléctrica (ENEE), Zamorano realiza y apoya investigaciones entomológicas que implican la colecta de insectos y otros artrópodos (arácnidos, milpiés, ciempiés) en varias regiones de Honduras, ante todo en áreas protegidas manejadas por el ICF. Los resultados son muy importantes para la obtención de conocimientos sobre la biodiversidad de Honduras y la estructura de sus ecosistemas únicos.

Para la gran mayoría de los parques nacionales y reservas biológicas de Honduras, todavía no existe un inventario confiable de las especies presentes de insectos o arácnidos; así, un monitoreo de artrópodos con métodos diferentes es una tarea muy importante para obtener datos sobre la riqueza biológica de estas áreas protegidas. Recientemente, por ejemplo, se realizaron investigaciones de la fauna entomológica en las áreas protegidas El Chile (2011), La Muralla (2011 y 2012), Celaque (2012), Misoco (2012), Patuca (2012) y cada año en Cerro Uyuca (una reserva biológica manejada por Zamorano), frecuentemente con el apoyo de estudiantes de Zamorano en sus pasantías, giras educativas o cursos. En todas estas investigaciones se aplican métodos diferentes de colecta para recolectar especímenes de gremios ecológicos, zonas y horas de actividad y microhábitats diferentes.

Los métodos más aplicados son trampas de caída (trampas tipo Barber), trampas amarillas (basadas en el efecto del color atractivo para muchos insectos voladores como polinizadores), instalación de lámparas de vapor de mercurio por la noche (que producen una parte considerable de luz ultravioleta (Figura 5), redes de mariposa, disección de madera y troncos en descomposición, y trampas de tipo Malaise (Six-Meter Malaise-Trap of Gressitt \& Gressitt). Con el último método mencionado, es posible recolectar miles de especímenes de insectos voladores (ante todo Hymenoptera, Diptera y Lepidoptera) en muy poco tiempo. Sin embargo, siempre hay que considerar el esfuerzo necesario de tiempo y trabajo para seleccionar y evaluar estas muestras, por eso normalmente hay que seleccionar algunas familias claves.

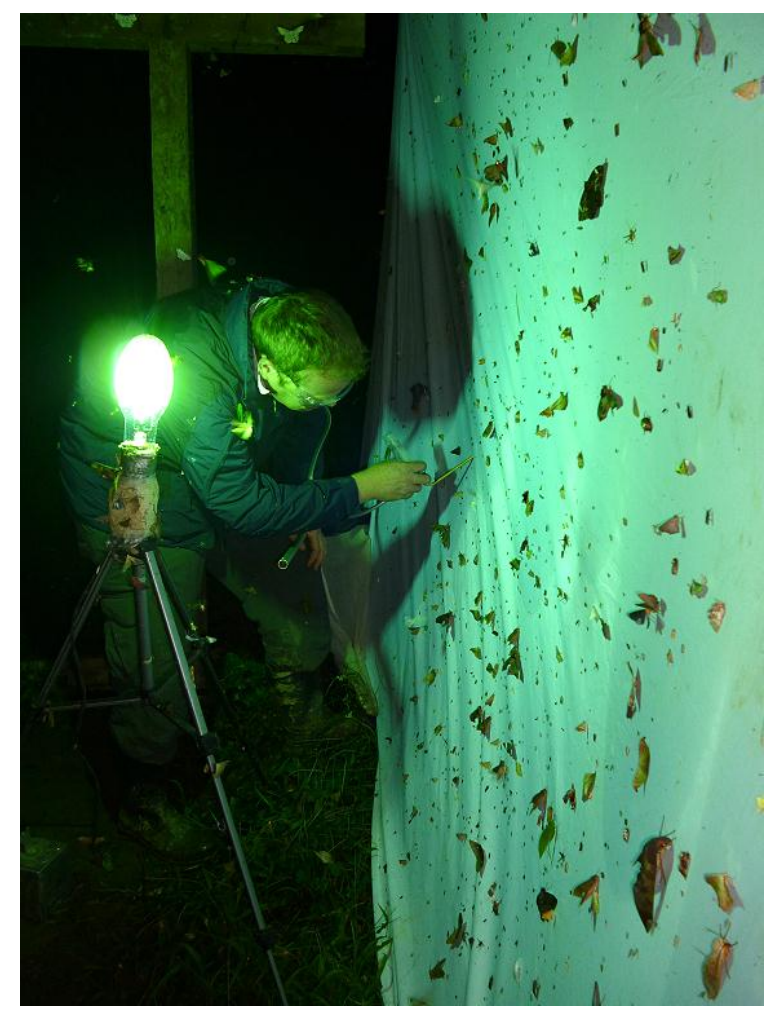

Figura 5. Colecta de himenópteros parasíticos con la luz de vapor de mercurio y un aspirador en el Refugio de Vida Silvestre La Muralla, Olancho, Honduras.

Para cada familia de insectos existen especialistas a nivel mundial, y porque no hay en Honduras entomólogos especializados, en la mayoría de casos, hay que enviar los especímenes a los especialistas, normalmente a los Estados Unidos, Canadá o Europa. Por eso, es difícil realizar inventarios de insectos y otros artrópodos a corto plazo, toma muchos meses y a 
veces años identificarlos hasta género y especie. Otro problema grave es la carencia de claves taxonómicas y específicas para la región, y específicamente para Honduras. Sin embargo, la Colección de Artrópodos de Zamorano ofrece muy buenas opciones para comparar especímenes recientemente recolectados con los conservados en la colección como referencia.

En la reserva biológica El Chile (Departamento de Francisco Morazán), antes no existía un inventario de las especies de artrópodos. En el 2011, como resultado de tres giras, 132 familias (clases Insecta y Arachnida) fueron recolectadas e identificadas; más de 1100 especímenes fueron evaluados e identificados (hasta familias, subfamilias, géneros y/o especies) y más de 800 especies (clases Insecta y Arachnida) fueron recolectadas y/o observadas; 214 fueron identificadas hasta géneros y especies (Moreno et al. 2011) (Figura 6). Se elaboran inventarios de los insectos y arácnidos de la reserva Misoco (Departamento de Francisco Morazán), completamente desconocidos, y del Parque Nacional Celaque (Departamento de Lempira), también es el primer inventario más detallado para este parque bien conocido e importante en el occidente de Honduras.

Otra colaboración nacional existe con la Empresa Nacional de Energía Eléctrica (ENEE), la cual está interesada en estudios de biodiversidad en sus embalses y represas. En este marco, se realizaron dos expediciones al embalse El Cajón (2010 y 2012), un área enorme que pertenece a tres departamentos de Honduras, Yoro, Comayagua y Cortés. Con el apoyo de Zamorano, el primer inventario detallado de los artrópodos del embalse fue elaborado; más de 120 familias (clase Insecta) fueron recolectadas e identificadas, más de 1500 especímenes (clase Insecta) fueron evaluados e identificados hasta familia, subfamilia, género y/o especie, y más de 425 especies de clases Insecta y Arachnida fueron recolectadas. En el 2012, se continuó esta colaboración con una primera investigación entomológica en la zona Patuca III, en áreas que se inundarán completamente en el 2014 por la construcción de la represa grande en el Río Patuca.

Existe una iniciativa nacional del ICF para investigar la biodiversidad de escarabajos estercoleros (Scarabaeidae: Scarabaeinae) como indicadores de la calidad e integridad ecológica de ecosistemas naturales de Honduras, ante todo de las áreas protegidas y bosque primarios de la zona norte del país. Zamorano colabora en la identificación de los especímenes recolectados, y la tesis de un estudiante del Departamento de Ambiente y Desarrollo de Zamorano, investigó la biodiversidad de esta subfamilia en el Parque Nacional Nombre de Dios, fue realizada como primer inventario de este taxón en el parque (Jut Solorzano 2012).

\section{Colaboraciones y Proyectos Internacionales Desde el 2008}

Desde el 2007, la Colección de Artrópodos de Zamorano pertenece al Centro Zamorano de Biodiversidad (CZB), manejado por el Departamento de Ambiente y Desarrollo. Cada año, varios equipos de científicos internacionales contactan al curador de la colección para sus investigaciones entomológicas con el apoyo de Zamorano como contraparte nacional y lugar para la colocación de especímenes duplicados, recolectados en sus expediciones. Zamorano apoya estas investigaciones y proyectos a través de negocios y mediación con el ICF, para facilitar los permisos de colecta en áreas protegidas y de exportación. Varios proyectos de gran importancia para los conocimientos sobre la biodiversidad de insectos de Honduras fueron realizados así exitosamente.

Zamorano, a través de la Colección de Artrópodos, fue la contraparte nacional del proyecto LLAMA (Leaf Litter Arthropods of Mesoamerica), un proyecto de cinco años de duración que investigaba la biodiversidad de las hormigas (Formicidae) y picudos (Curculionidae) que habitan la hojarasca de los bosques naturales a tres altitudes (nivel bajo, mediano y bosque nublado). Se tomaron muestras en México (Chiapas), Guatemala, Honduras y Nicaragua. En el 2010 y 2011, el taller del proyecto para clasificar las muestras obtenidas fue realizado en la Colección de Artrópodos de Zamorano. En el 2010, la expedición del proyecto tomó muestras en nueve parques nacionales y reservas biológicas de Honduras. Con los resultados se han descrito varias especies nuevas para la ciencia, y se han escrito revisiones sistemáticas de géneros de picudos y hormigas (Anderson 2010, Longino 2012). 


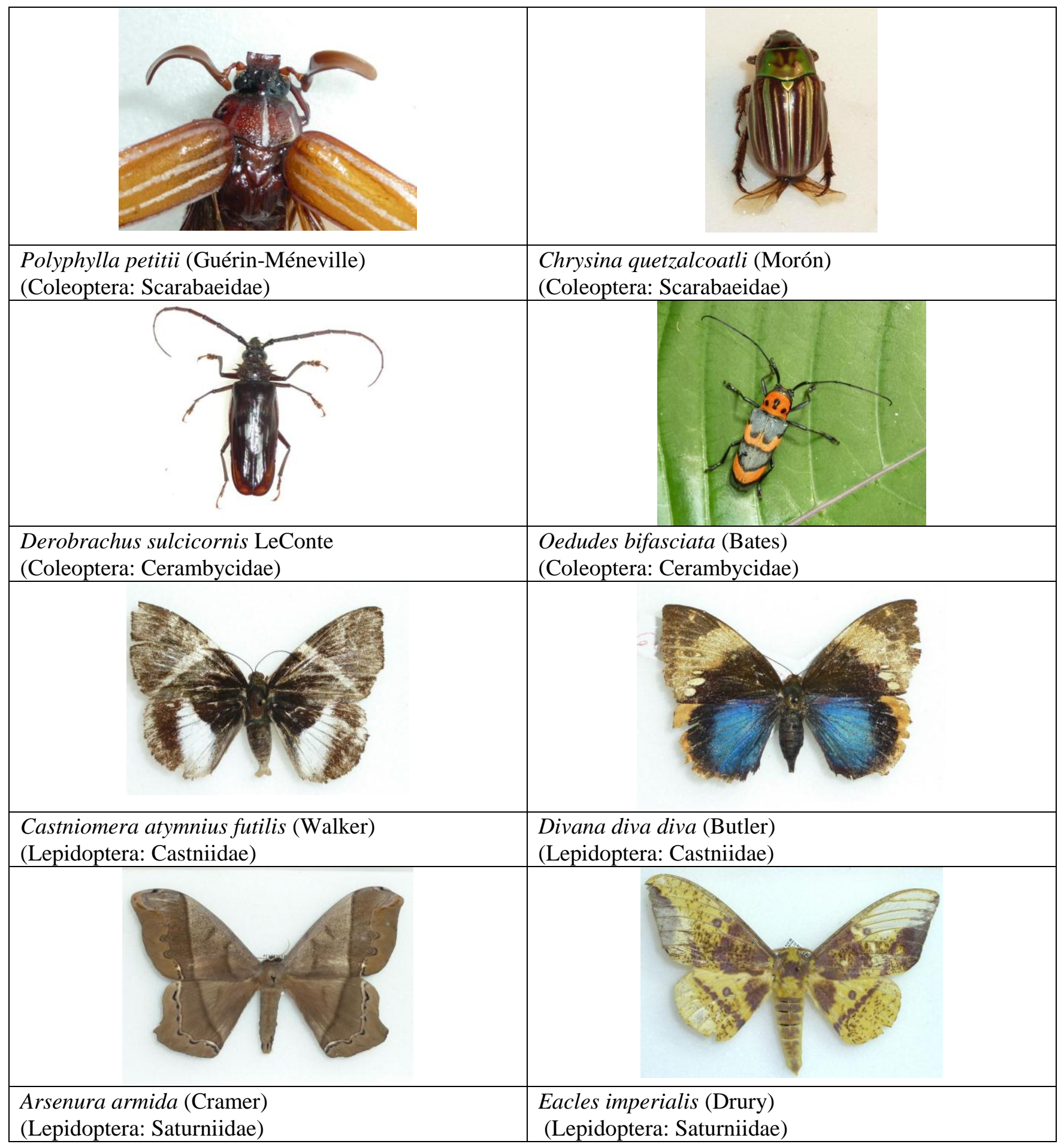

Figura 6. Ejemplos de especímenes recolectados en el marco del primer inventario entomológico de la reserva biológica El Chile (Francisco Morazán, Honduras, agosto-octubre de 2011, colaboración con el ICF Guaimaca). 
Otra colaboración internacional de gran importancia existe con el McGuire Center for Lepidoptera and Biodiversity (Gainesville, Florida, Estados Unidos) desde el año 2009. Científicos del centro, liderados por la Dra. Jacqueline Y. Miller, investigan cada año la biodiversidad de mariposas y polillas de Honduras, con colectas intensivas en la zona alrededor y dentro del Parque Nacional Pico Bonito (Departamento de Atlántida). El objetivo principal del proyecto es inventariar la biodiversidad de lepidópteros en una forma completa e integrada, así como obtener datos de referencia cualitativos y cuantitativos de los grupos bioindicadores dentro del orden Lepidoptera, en cinco sitios en Honduras. Los esfuerzos de la investigación se centran en la diversidad de especies, abundancia, fenología y requerimientos de hábitat con las observaciones registradas en la historia de vida y las asociaciones de plantas huésped. Se han donado e incorporado muchos especímenes duplicados de esta investigación a la Colección de Artrópodos de Zamorano, y los científicos elaboraron un primer inventario de los lepidópteros de Honduras (Miller et al. 2012).

Desde el 2012, Zamorano mantiene una colaboración con el Museo de Historia Natural de Stuttgart, Alemania, en el marco del proyecto Biodiversidad de los Himenópteros Parasíticos de Honduras (líder: Dr. Lars Krogmann). Las primeras colectas intensivas fueron en el 2012 en las áreas protegidas de Misoco, La Muralla, Uyuca y Patuca, y miles de especímenes recolectados (ante todo de la superfamilia Chalcidoidea) están identificados hasta género y especie. Es muy probable que varias especies nuevas de parasitoides resulten de esta investigación, la cual se continuará durante los próximos años.

Finalmente, Zamorano funciona como una de las contrapartes nacionales del proyecto Biodiversidad y Endemismo en Bosques Nublados Desconocidos del Noreste de Honduras (2012-2013), financiado por National Geographic, y un componente importante de esta investigación son las familias claves de coleópteros (ante todo Elateridae, Scarabaeidae, Passalidae) las cuales fueron recolectadas en el 2012 en seis zonas (áreas protegidas y zonas naturales pendientes de una protección por la ley) ubicadas en los departamentos de Olancho y Atlántida. La identificación de las especies obtenidas está elaborada, y la evaluación de estas familias claves probablemente descubrirá no solamente especies nuevas, endémicas o registros nuevos para Honduras, pero también información científica sobre el valor, la integridad ecológica, y la unicidad de los sitios investigados.

\section{Perspectivas y Conclusiones}

Los conocimientos sobre la riqueza de la biodiversidad de los insectos aún están en un estado inmaduro en Honduras. Sin embargo, se pueden destacar los avances que se han hecho con las publicaciones de catálogos de las especies de Odonata (Dunkle 1988), Tenuipalpidae (Evans et al. 1993), Pentatomidae (Arismendi y Thomas 2003), Cerambycidae (Turnbow et al. 2003b), Bruchinae (Turnbow et al. 2003a), Dynastinae (Ratcliffe y Cave 2006), Aphididae (Evans y Halbert 2007), Sphingidae (Samayoa y Cave 2008) y Lepidoptera (Miller et al. 2012). Todos estos estudios utilizaron especímenes y sus datos asociados que están alojados en la Colección de Artrópodos de Zamorano.

En Honduras existen otras colecciones importantes como el Museo de Entomología de la Universidad Nacional Autónoma de Honduras (UNAH), el Museo de Mariposas de La Ceiba, cuyo dueño es Robert Lehman, y las colecciones de la Escuela Nacional de Ciencias Forestales y el Centro Universitario de la Región Litoral Atlántica. Juntas, todas las colecciones y sus curadores podrán contribuir con la investigación de la biodiversidad de artrópodos en Honduras. Se ha creado un portal para unir las colecciones científicas de Centroamérica, llamado bioSIAM (Portal de Biodiversidad del Sistema de Información Ambiental Mesoamericano), una iniciativa regional en el marco del programa PROMEBIO, financiado por la CCAD (Comisión Centroamericana de Ambiente y Desarrollo). Una presentación virtual de la Colección de Artrópodos de Zamorano a través de este portal fue elaborada y hay esfuerzos para actualizar y conectar la base de datos respectiva a esta página web, facilitando acceso a la base de datos desde fuera de Zamorano.

La Colección de Artrópodos de Zamorano siempre ofrece muchas posibilidades para las tesis, pasantías y prácticas de los estudiantes de Zamorano. Por ejemplo, sobre la biodiversidad, taxonomía, 
sistemática, biogeografía y ecología de los artrópodos. La colección apoya a la enseñanza de las clases de entomología de Zamorano (Figura 7) y el laboratorio de entomología (Carrera de Ingeniería Agronómica, tercer año) o el módulo Biodiversidad y Ecología de Insectos (Carrera de Ingeniería en Ambiente y Desarrollo, segundo año). Frecuentemente, guías regulares y especiales para grupos de estudiantes, alumnos, científicos, visitantes académicos y el público se realizan en la colección con mucho éxito. Durante el 2011 y 2012, se realizaron cinco exhibiciones en el campus de Zamorano y fuera de Zamorano, asistieron miles de visitantes que quedaron emocionados al conocer la biodiversidad de los artrópodos de Honduras. Las exhibiciones fueron con cajas llamativas de la colección y arácnidos e insectos vivos colocados en terrarios que forman la base para un insectario permanente, planificado para el 2013.

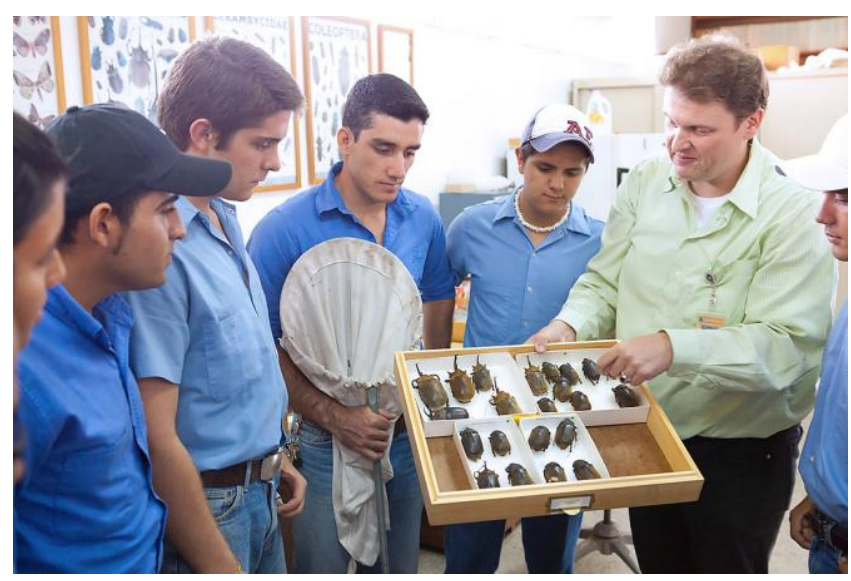

Figura 7. Enseñanza de entomología en la Colección de Artrópodos, con estudiantes de la Escuela Agrícola Panamericana, Zamorano, Honduras.

La colección incluye miles de especímenes de plagas que tienen gran importancia económica en la agricultura y la economía forestal. El manejo de los cultivos necesita una identificación exacta de las especies de plagas para encontrar estrategias y métodos de control biológico y del manejo integrado de plagas; por ejemplo, la liberación de depredadores, parasitoides y otros enemigos naturales. Por comparación con las especies identificadas en la colección, un diagnóstico de muestras de plagas hasta género o especie a menudo es posible. Recientemente, una colaboración con OIRSA (Organismo Internacional Regional de Sanidad Agropecuaria) en diagnóstico de plagas invasivas, fue establecida, y se capacitaron a empleados y técnicos de OIRSA en el inicio y mantenimiento de colecciones entomológicas y la identificación de ciertos grupos de plagas invasivas.

Durante los próximos años, la Colección de Artrópodos de Zamorano recibirá otra colección de gran importancia, la colección del Museo Entomológico de León, Nicaragua. Esta donación es del Dr. Jean-Michel Maes, uno de los entomólogos más conocidos de Nicaragua, quien ha recolectado por más de 30 años en Nicaragua y publicó el Catálogo de los Insectos y Artrópodos Terrestres de Nicaragua (Maes 1998), una obra sumamente importante y única. Su colección comprende más de 250,000 especímenes, colectados principalmente en Nicaragua. En un proceso extensivo de varios años, esta colección de gran importancia en conocimientos entomológicos de Nicaragua y toda la región, será incorporada a la colección de Zamorano, con la meta de unir los especímenes de ambas colecciones en un solo sistema taxonómico y crear una base de datos que incluye los especímenes de Honduras y Nicaragua.

Varios miles de especímenes (ante todo familias del orden Coleoptera) del Museo Entomológico de León, Nicaragua, ya fueron incorporados a la Colección de Artrópodos de Zamorano, y por una donación de la institución Alemana CIM (Centro para la Migración Internacional y el Desarrollo) la cual pertenece a la GIZ (Sociedad Alemana para la Cooperación Internacional), y fondos propios del Departamento de Ciencia y Producción Agropecuaria de Zamorano, 10 gabinetes nuevos y 250 cajas entomológicas fueron adquiridos en el 2011, facilitando espacio para colocar una gran parte de la colección del Museo Entomológico. Por lo tanto, la colección de Zamorano será un conglomerado de casi medio millón de insectos y la más importante y representativa de Honduras y Nicaragua, además, una de las colecciones más grandes de Centroamérica, junto con las del Smithsonian Tropical Research Institute en Panamá y el Instituto Nacional de Biodiversidad en Costa Rica. 


\section{Literatura Citada}

Anderson, R.S. (2010). A taxonomic monograph of the Middle-American leaf-litter inhabiting weevil genus Theognete Champion (Coleoptera: Curculionidae; Molytinae; Lymantini). Zootaxa 2458: 1-127.

Arismendi, N. y D.B. Thomas. 2003. Pentatomidae (Heteroptera) of Honduras: a checklist with description of a new ochlerine genus. Insecta Mundi 17: 219-236.

Cave, R.D. 2001. Jewel scarabs. National Geographic Magazine 199(2): 52-61.

Cave, R.D. y R. J. Cordero. 2002. Insectos de Honduras. Escuela Agrícola Panamericana, Zamorano, Honduras.

Cave, R.D., R.J. Cordero, y G. A. Peña. 2001. La Vida Fascinante y Exitosa de los Insectos. Zamorano Academic Press, Zamorano, Honduras. 60 p.

Dunkle, S. 1988. A list of the Odonata of Honduras. Ceiba 29: 41-49.

Evans, G.A., H.L. Cromroy y R. Ochoa. 1993. The Tenuipalpidae of Honduras. Florida Entomologist 76: 126-155.

Evans, G.A. y S.E. Halbert. 2007. A checklist of the aphids of Honduras (Hemiptera: Aphididae). Florida Entomologist 90: 518-523.

Hawks, D.C., and G.P. Bruyea. 1999. Chrysina cavei, a new species from Honduras (Coleoptera: Scarabaeidae: Rutelinae). Consortium Coleopterorum 3: 15-20.

Jut Solorzano, J.C. 2012. Comparación de la biodiversidad de escarabajos estercoleros (Scarabaeidae: Scarabaeinae) en diferentes ecosistemas en el parque nacional Nombre de Dios, Atlántida, Honduras. Tesis Ing. Agr. Escuela Agrícola Panamericana, El Zamorano, Honduras.
Longino, J.T. 2012. A review of the ant genus Adelomyrmex Emery 1897 (Hymenoptera, Formicidae) in Central America. Zootaxa 3456: 1-35.

Maes, J.M. 1998. Catálogo de los insectos y artrópodos terrestres de Nicaragua. Volumen I-III. Imprenta PRINT-León, Nicaragua. 1881 p.

Miller, J.Y., D.L. Matthews, A.D. Warren, M.A. Solis, D.J. Harvey, P. Gentili-Poole, R. Lehman, T.C. Emmel y C.V. Covell, Jr. 2012. An annotated list of the Lepidoptera of Honduras. Insecta Mundi 0205: 1-72.

Moreno, R., Vásquez, S. \& Palacios, A. (eds.). 2011. Segundo estudio de biodiversidad en la reserva biológica "El Chile". Informe del ICF Oficina Local Guaimaca. 62 p.

Ratcliffe, B.C. y R.D. Cave. 2006. The Dynastine scarab beetles of Honduras, Nicaragua, and El Salvador. Bulletin of the University of Nebraska State Museum 21: $1-424$

Samayoa, A.C. y R.D. Cave. 2008. Catálogo de las Especies de Sphingidae (Lepidoptera) en Honduras. Ceiba 49: 103-117.

Turnbow, R.H., R.D. Cave y J.M. Kingsolver. 2003a. An annotated checklist of the Bruchidae of Honduras. Ceiba 44: 269-278.

Turnbow, R.H., Jr., R.D. Cave y M.C. Thomas. 2003b. A list of the Cerambycidae of Honduras, with additions of previously unrecorded species. Ceiba 44: 1-43.

Recibido para publicación el 3 de diciembre de 2012.

Aceptado para publicación el 29 de enero de 2013 